\title{
Influência Exercida pelos Instrumentos de Intervenção do Banco Central do Brasil sobre a Volatilidade Cambial
}

\author{
Adilson Giovanini ${ }^{1}$
}

Roberto Meurer ${ }^{2}$

Resumo: O objetivo deste trabalho é verificar quais os instrumentos utilizados e os efeitos da intervenção do Banco Central sobre a taxa e a volatilidade do câmbio no período entre 14/12/2006 e 02/12/2014. Para isto são estimados dois modelos VAR, sem e com expectativas, para verificar se a volatilidade das expectativas dos agentes e as intervenções do BCB influenciam a volatilidade do câmbio. O modelo VAR com expectativas possui um poder de explicação maior que o modelo que não considera as expectativas. Os coeficientes estimados para o modelo VAR são significativos, evidenciando que os instrumentos utilizados pelo BCB influenciam a taxa de câmbio. Os resultados para o período 14/12/2006 - 28/05/2009 mostraram que o desvio do câmbio em relação às expectativas e o desvio dos juros em relação às expectativas explicaram o retorno do câmbio, o qual também é explicado pelas intervenções do BCB através de swap cambial e mercado a vista.

Palavras chaves: câmbio; expectativas; intervenções do BCB

JEL: Co1, C13, E43

1 UFSC.

2 UFSC. 
GIOVANINI, A.; MEURER, R. A Influência Exercida pelos Instrumentos de Intervenção do Banco Central do Brasil....

Abstract: The objective of this work is to verify which are the instruments used and the effects of Central Bank intervention on the rate foreign exchange volatility in the period between 14/12/2006 and 12/02/2014. For this are estimated two VAR models, with and without expectations, to verify that the volatility of agents' expectations and the $B C B$ interventions influence the exchange rate volatility. The VAR model with expectations has a power greater explanation that the model that does not consider the expectations. The coefficients estimated for the VAR model are significant, showing that the instruments used by BCB influence the exchange rate. The results for the period 14/12/2006 - 28/05/2009 showed that the exchange rate deviation from expectations and the diversion of interest relative to expectations explain the return of the exchange, which is also explained by the interventions of the BCB through a currency swap and the spot market.

Keywords: Exchange; expectations; operations of the BCB

JEL: Co1, C13, E43

\section{Introdução}

Após a adoção do regime de câmbio flutuante pelo Brasil, em 1999, o Banco Central do Brasil (BCB) continuou interferindo no mercado cambial através de diversos instrumentos, com o objetivo de diminuir a volatilidade da taxa de câmbio. Estas intervenções do BCB podem reduzir os efeitos desfavoráveis que a volatilidade cambial exerce sobre importadores, exportadores, investidores estrangeiros e consumidores. As intervenções possuem o objetivo de prover liquidez em momentos de necessidade ou acumular reservas, mas não influenciar no nível ou na tendência do câmbio (Bastos E Fontes, 2013).

Algumas justificativas para a intervenção do banco central no câmbio são encontradas em Obstfeld (1988) e Rogoff (1984), segundo os quais a volatilidade do câmbio, associada à instabilidade das expectativas pode levar os agentes econômicos a apresentarem uma perda significativa de bem-estar, que se torna ainda mais elevada na presença de aversão ao risco ou caso o país possua uma elevada dependência do mercado externo. A flutuação no câmbio leva a uma flutuação da capacidade de importação e exportação de bens, o que resulta em uma flutuação do nível de consumo.

Os principais instrumentos utilizados pelo BCB para intervir no câmbio no período 2007-2013 foram a taxa de juros, as intervenções no mercado a vista, a alíquota do IOF, o mercado a termo, as linhas de crédito e as intervenções via swap cambial.

Os únicos estudos que identificaram a influência que os instrumentos de intervenção utilizados pelo BCB possuem sobre o câmbio, discriminando os instrumentos segundo o tipo de intervenção, foram os estudos realizados por Oliveira e Plaga (2011) e por Meurer et al. (2010). Estes estudos não levaram 
GIOVANINI, A.; MEURER, R. A Influência Exercida pelos Instrumentos de Intervenção do Banco Central do Brasil....

em consideração a influência que as expectativas de câmbio e de taxa juro dos agentes exercem sobre os instrumentos de intervenção do BCB. Este artigo busca suprir esta lacuna, tendo como objetivo analisar o modo como os instrumentos de intervenção utilizados pelo BCB influencia a taxa de câmbio nominal. Para isto a influência que cada instrumento de intervenção exerce sobre o câmbio é identificada através da estimação de um modelo VAR e a influência sobre a volatilidade é identificada através de um modelo BEKK.

Em um primeiro momento estimaram-se regressões para o modelo que não inclui as expectativas dos agentes. Os resultados encontrados para oforecast encompassing test, para o CUSUM e para o CUSUMSQ indicam que a crise do subprime provocou uma quebra estrutural na taxa de juros e na taxa de câmbio. Por isto, estimam-se dois modelos VAR, para 14/12/2006-01/o4/2008 e 02/04/2008-02/12/2014. Posteriormente, são estimadas regressões, incluindo-se o desvio da taxa de juros e do câmbio em relação às expectativas dos agentes, os testes para a presença de quebra estrutural indicaram que a amostra deveria ser dividida em três subamostras, estimando-se três modelos VAR, um para o período que antecede a quebra estrutural, 14/12/2006 - 28/05/2009, outro para o período 29/05/2009 - 12/01/2012 e outro para o período 13/01/2012 - 30/o8/2013. No primeiro período, o retorno do câmbio é explicado apenas pelo câmbio defasado e pelo desvio do câmbio e da taxa de juros em relação às expectativas dos agentes, no segundo período, as intervenções via swap passam a influenciar no retorno apresentado pelo câmbio e no terceiro período as intervenções no mercado a vista passam a influenciar no câmbio, enquanto que as intervenções via swap deixam ser significativas.

$\mathrm{O}$ artigo se encontra dividido conforme segue. A seção 1 apresenta os instrumentos utilizados pelo BCB para intervir no câmbio. A seção 2 apresenta as principais evidências encontradas na literatura econômica sobre a influência que o BCB exerce sobre o câmbio. A seção 3 apresenta a metodologia utilizada para identificar o modo como o BCB influencia na cotação do câmbio. A seção 4 mostra os resultados encontrados e na última seção são feitas as considerações finais.

\section{Instrumentos Utilizados pelo BCB para Intervir no Câmbio}

O BCB intervém no câmbio através da taxa de juros, IOF, mercado a vista, títulos públicos indexados ao dólar, swap cambial e swap reverso. O aumento da taxa de juros, dada a paridade de juros a descoberto, tem como objetivo atrair capitais estrangeiros e elevar o custo de carregamento da moeda estrangeira 
GIOVANINI, A.; MEURER, R. A Influência Exercida pelos Instrumentos de Intervenção do Banco Central do Brasil....

pelos especuladores, suavizando a depreciação. Por outro lado, a diminuição da taxa de juros torna os ativos domésticos menos atrativos, resultando na saída de capital e na depreciação da moeda doméstica (Merlin, 2002).

A intervenção no mercado de câmbio também pode ser feita através da compra ou venda de dólares no mercado à vista. A limitação apresentada pelas intervenções à vista é que elas requerem que as reservas internacionais estejam em um nível adequado, o que limita a utilização deste instrumento. Na presença de choques sobre o câmbio, a incerteza quanto à capacidade $\mathrm{e}$ credibilidade do BCB pode fazer com que as reservas internacionais sejam consumidas rapidamente (Oliveira e Plaga, 2011).

A partir de março de 2002 o BCB começou a intervir no câmbio através da emissão de swap cambial. Nessa forma de intervenção o BCB assume a posição comprada do referido contrato, na ponta cambial, e as instituições assumem a posição vendida na ponta juros DI (Resolução 2.939, BCB). Se a desvalorização cambial for maior que o juro DI, o BCB paga a diferença às instituições, caso contrário, recebe. Por outro lado, no swap reverso, criado em 2005, o BCB assume a ponta DI e as instituições contratantes assumem a ponta cambial. Nesse tipo de contrato, se a desvalorização cambial for maior que o juro DI o BCB recebe a diferença das instituições e se a desvalorização cambial for menor que o juro DI o BCB efetua o pagamento (Viola et al, 2009).

Segundo Silva e Resende (2010) e Carvalho e Garcia (2006), o IOF era uma dos principais instrumentos utilizados pelo BCB para controlar o influxo de capitais na década de 1990. A partir de 14 de dezembro de 2007, Decreto ${ }^{\circ}$ 6.306, o governo voltou a utilizar a cobrança de IOF sobre o ingresso e a saída de capital estrangeiro como um instrumento adicional de intervenção cambial. A alíquota passou de $0 \%$ para 1,5\% no dia 12 de março de 2008, Decreto ${ }^{0}$ 6.391, e para 6\% em 04 de setembro de 2010, decreto 7412. Em o5 de junho de 2013, decreto 8023, a alíquota do IOF foi revista, sendo zerada.

\section{Evidências Empíricas}

Oliveira e Plaga (2011) utilizaram o modelo E-GARCH de Nelson e Cao (1992) para modelar a volatilidade condicional da taxa de câmbio a partir de uma base composta por dados diários da taxa de câmbio nominal e das intervenções realizadas pelo BCB, discriminada por tipo de intervenção realizada no período entre janeiro de 1999 e setembro de 2006. O único instrumento de intervenção que conseguiu diminuir a volatilidade condicional da taxa de câmbio nas crises cambiais foram os títulos cambiais. As intervenções no mercado à vista adicionaram volatilidade na crise cambial de 1999 e dimi- 
GIOVANINI, A.; MEURER, R. A Influência Exercida pelos Instrumentos de Intervenção do Banco Central do Brasil....

nuíram a volatilidade na crise de 2002. Um resultado interessante é que a taxa de juros não conseguiu reduzir a volatilidade nos períodos de crise. Por outro lado, fora dos períodos de crise cambial, o BCB foi eficaz, influenciou a volatilidade condicional da taxa de câmbio através dos títulos cambiais e da taxa de juros.

Segundo Meurer et al. (2010) e Araújo e Goldfajn (2004), as intervenções realizadas de forma contínua afetaram mais o comportamento do câmbio do que as intervenções realizadas de forma isolada, causando ajustes mais precisos nas expectativas dos agentes do que as intervenções pontuais. Meurer et al. (2010) analisaram os instrumentos utilizados pelo BCB para intervir no câmbio no período 1999-2008. As intervenções no mercado à vista possuem efeito sobre o câmbio, principalmente nos dias seguintes às intervenções. As intervenções com títulos e swaps também influenciam o câmbio, porém com uma defasagem elevada. O modelo EGARCH indicou uma assimetria de choques na volatilidade, intervenções relacionadas a depreciações apresentam maior efeito na volatilidade que as intervenções vinculadas a apreciações (Meurer et al., 2010).

Viola et al. (2009) analisaram o impacto que as ofertas de swap cambial e swap cambial reverso realizadas pelo BCB têm sobre a estrutura a termo do cupom cambial entre 02/01/2003 e 30/09/2008. Os resultados encontrados indicam que o swap cambial influencia na curva de cupom cambial (juro pago nos títulos indexados ao dólar, dado pela diferença entre a taxa de juros interna e a desvalorização da taxa de câmbio) e na volatilidade do dólar à vista na direção prevista.

Munhoz (2013) recorre a um modelo VAR para identificar se a cobrança de IOF influenciou no fluxo de capitais estrangeiros no período entre janeiro de 1995 e dezembro de 2011. Os resultados indicam que o IOF não representou uma barreira à movimentação de capitais. A taxação via IOF não foi suficiente para deter a trajetória de apreciação do real via entrada de capitais, os quais estão mais intimamente associados a movimentos da taxa de câmbio e do risco-país.

Sicsú (2002) utiliza análise gráfica para identificar qual a relação existente entre a taxa Selic e a taxa de câmbio no período 1999-2002. Os resultados encontrados indicam que o BCB utilizou a taxa de juros para diminuir a volatilidade cambial.

Em um regime de metas de inflação em que o banco central segue uma regra de Taylor, uma depreciação da moeda local levará a um aumento da taxa de juros. A consequência disto é que um regime de câmbio flutuante acentua a inter-relação existente entre a taxa de juros e a taxa de câmbio, aumentando a influência que as decisões de portfólio dos investidores globais exercem sobre esses preços-chave (Merlin, 2002). Conforme observado por Cerqueira (2011), a taxa de juros e a variância da taxa de câmbio real efetiva não são 
GIOVANINI, A.; MEURER, R. A Influência Exercida pelos Instrumentos de Intervenção do Banco Central do Brasil....

independentes, a volatilidade cambial no Brasil pode estar associada à regra de política monetária adotada no país. As economias que possuem algum controle sobre os fluxos de capitais conseguem, pelo menos parcialmente, diminuir a influência que os ciclos cambiais exercem sobre as políticas monetária e cambial (Mohanty e Scatigna, 2005).

Gonçalves e Guimarães (2011) testa a hipótese de que em um país onde a probabilidade de calote é alta, aumentos da taxa de juro podem gerar depreciação da moeda local. Os resultados corroboram com esta hipótese, no período 2000/2003 elevações inesperadas dos juros do Brasil geraram em média depreciações da taxa nominal de câmbio.

Por fim, Toledo (2002) apresenta uma visão diferente sobre a influência que a taxa de juros possui sobre o câmbio, segundo o qual a taxa de juros influencia na percepção de risco dos investidores estrangeiros. Quando os juros aumentam, os investidores não são atraídos pela remuneração mais elevada que podem auferir ao investir no mercado brasileiro. O primeiro fator que eles levam em consideração na hora de realocar o seu portfólio não é o retorno que vão auferir, mas a estabilidade dos fundamentos macroeconômicos. $\mathrm{O}$ aumento dos juros, apesar de resultar em um retorno mais elevado resulta em uma maior fragilidade macroeconômica, aumentando a percepção de risco dos investidores.

\section{Metodologia}

3.1 Dados

A Tabela 1 apresenta as variáveis utilizadas e suas fontes. Os dados utilizados possuem frequência diária e foram obtidos para o período entre 14/12/2006 e 02/12/2014, para o qual o BCB disponibiliza dados sobre as intervenções à vista e via swap. 
GIOVANINI, A.; MEURER, R. A Influência Exercida pelos Instrumentos de Intervenção do Banco Central do Brasil....

TABELA 1 - DESCRIÇÃO DAS VARIÁVEIS UTILIZADAS

\begin{tabular}{|c|c|c|c|}
\hline Símbolo & Descrição & Fonte & Unidade \\
\hline $\mathrm{S}_{\mathrm{t}}$ & Taxa de câmbio & $\begin{array}{l}\text { BCB-SGS*- Sé- } \\
\text { rie } 7381\end{array}$ & $\mathrm{R} \$ / \mathrm{US} \$$ \\
\hline $\mathbf{i}_{t}$ & Taxa de Juros & $\begin{array}{c}\text { BCB-SGS- Série } \\
11\end{array}$ & $\begin{array}{c}\text { Percentual ao } \\
\text { ano }\end{array}$ \\
\hline $\mathrm{I}_{\mathrm{t}}^{\text {Vist }}$ & Intervenções à vista & $\begin{array}{c}\text { BCB-SGS- Série } \\
1789\end{array}$ & Bilhões R\$ \\
\hline$I_{t}^{\text {Swap }}$ & $\begin{array}{l}\text { Intervenções via swap e } \\
\text { swap reverso }\end{array}$ & $\mathrm{BCB}^{* *}$ & $\begin{array}{l}100 \text { milhões } \\
\mathrm{R} \$\end{array}$ \\
\hline $\mathrm{I}_{\mathrm{t}}^{\text {Titu }}$ & $\begin{array}{c}\text { Intervenções via títulos } \\
\text { cambiais }\end{array}$ & $\begin{array}{l}\text { BCB-SGS- Série } \\
10604 \text { e } 10720\end{array}$ & Milhões R\$ \\
\hline$S_{t, e}$ & $\begin{array}{l}\text { Expectativa de Câmbio do } \\
\text { mercado um mês à frente }\end{array}$ & BCB-SGS & $\mathrm{R} \$ / \mathrm{US} \$$ \\
\hline $\mathbf{s}_{\mathrm{t}}^{\mathrm{e}}$ & $\begin{array}{l}\text { Desvio da taxa de câmbio } \\
\text { em relação às expectativas } \\
\text { dos agentes ( }\end{array}$ & & $\mathrm{R} \$ / \mathrm{US} \$$ \\
\hline $\mathrm{IOF}_{1}$ & $\begin{array}{c}\text { Dummy para o período } \\
\text { 12/o3/2008 - 03/o9/2010 } \\
(\text { IOF }=0,5 \%)\end{array}$ & Notas do BCB & Percentual \\
\hline $\mathrm{IOF}_{2}$ & $\begin{array}{c}\text { Dummy para o período } \\
\text { 04/09/2010 - 05/07/2013 } \\
(\text { IOF }=6 \%)\end{array}$ & Notas do BCB & Percentual \\
\hline$i_{t}^{e}$ & $\begin{array}{l}\text { Desvio da taxa de juros em } \\
\text { relação às expectativas do } \\
\text { mercado () }\end{array}$ & & Percentual \\
\hline $\mathrm{i}_{\mathrm{t}, \mathrm{e}}$ & $\begin{array}{l}\text { Expectativa de juros do } \\
\text { mercado um mês à frente }\end{array}$ & BCB-SGS & Percentual \\
\hline
\end{tabular}

Fonte: Elaboração própria, " $S G S$ - Sistema Gerenciador de Séries Temporais - BCB, ${ }^{* *}$ Departamento de Mercado Aberto

Os dados referentes à intervenção via mercado à vista são disponibilizados pelo BACEN na série Condicionantes da Base Monetária - Conta Operações com o Setor Externo, que além das intervenções diretas, inclui outras operações, como trânsito de recursos de organismos internacionais destinados à manutenção de suas representações no Brasil e projetos de saneamento básico.

O Desvio da taxa de juros em relação às expectativas do mercado, $\mathrm{i}_{\mathrm{t}}^{\mathrm{e}}, \mathrm{e}$ o desvio da taxa de câmbio em relação às expectativas são definidos como: 
GIOVANINI, A.; MEURER, R. A Influência Exercida pelos Instrumentos de Intervenção do Banco Central do Brasil....

$$
\begin{aligned}
& i_{t}^{e}=i_{t, e}-i_{t}, \\
& s_{t}^{e}=s_{t, e}-s_{t},
\end{aligned}
$$

em que, $i_{t, e}$ é a expectativa de juros do mercado um mês à frente, $i_{t}$ é a taxa de juros atual, $\mathbf{s}_{t, \mathrm{e}}$ é a expectativa de câmbio do mercado um mês à frente $\mathrm{e}$ $\mathrm{S}_{\mathrm{t}}$ é a taxa de câmbio atual.

A variável $\mathbf{S}_{\mathrm{t}}^{\mathrm{e}}$ possui duas características importantes, que merecem ser destacadas. 1) O sinal de $S_{t}^{e}$ identifica o comportamento dos agentes. Nos períodos em que o sinal é positivo a expectativa de câmbio dos agentes, $\mathrm{S}_{\mathrm{t}, \mathrm{e}}$ é superior ao câmbio observado, $\mathrm{S}_{\mathrm{t}}$, de modo que eles consideram que a taxa de câmbio se elevará. Por outro lado, quando o sinal de $\mathbf{S}_{\mathrm{t}}^{\mathrm{e}}$ é negativo, a expectativa de taxa de câmbio dos agentes, $\mathbf{S}_{\mathrm{t}, \mathrm{e}}$, é inferior à taxa de câmbio observada, $\mathbf{S}_{\mathrm{t}}$, de modo que eles consideram que a taxa de câmbio vai se reduzir. 2) A magnitude do desvio das expectativas, $\mathrm{s}_{\mathrm{t}}^{\mathrm{e}}$, identifica o quanto a expectativa dos agentes é inferior ou superior a taxa de câmbio observada. Esta é a grande vantagem desta variável, uma vez que ela, além de identificar se a expectativa dos agentes é de aumento ou diminuição na taxa de câmbio, também mensura a diferençaentre as expectativas dos agentes e o valor observado no mercado ${ }^{1}$. Estas características também são válidas para $i_{t}^{e}$.

\subsection{Modelagem}

O referencial usado para a realização deste estudo é o modelo empírico que analisa a mudança no câmbio, proposto por Oliveira e Plaga (2011):

$$
\Delta \mathrm{S}_{\mathrm{t}}=\beta_{\mathrm{o}}+\beta_{1} \Delta \mathrm{S}_{\mathrm{t}-1}+\beta_{2} \Delta \mathrm{i}_{\mathrm{t}}+\beta_{3} \mathrm{I}_{\mathrm{t}}^{\mathrm{Vist}}+\beta_{4} \mathrm{I}_{\mathrm{t}}^{\mathrm{Swap}}+\beta_{5} \mathrm{I}_{\mathrm{t}}^{\mathrm{Titu}}+\varepsilon_{\mathrm{t}}
$$

o modelo foi adaptado para o presente problema de pesquisa, podendo ser expresso através do seguinte modelo VAR:

\footnotetext{
1.Uma alternativa ao desvio das expectativas é a variação do desvio das expectativas, $i_{t}^{e}=\left(i_{t, e}-i_{t-1, e}\right) /\left(i_{t}-i_{t-1}\right)$. Todavia, esta variável identifica apenas a velocidade de ajuste das expectativas dos agentes, perdendo informação sobre a magnitude do desvio das expectativas dos agentes em relação ao valor de mercado, informação considerada relevante neste estudo.
} 
GIOVANINI, A.; MEURER, R. A Influência Exercida pelos Instrumentos de Intervenção do Banco Central do Brasil...

$$
\Delta s_{t}=\alpha_{0}+\sum_{\mathrm{k}=1}^{\mathrm{p}} \alpha_{k} \Delta s_{t-k}+\sum_{\mathrm{k}=1}^{\mathrm{p}} \beta_{k} I_{t-k}+\sum_{\mathrm{k}=1}^{\mathrm{p}} \gamma_{k} I O F_{1, t-k}+\sum_{\mathrm{t}=1}^{\mathrm{p}} \gamma_{k} I O F_{2, t-k} \gamma_{k}+\varepsilon_{t}
$$

Como alternativa ao modelo da equação (2) considerou-se a influência que as expectativas dos agentes possuem sobre a volatilidade cambial. Para isto substitui-se a taxa de câmbio e a taxa de juros pelo desvio em relação as expectativa dos agentes.

A influência exercida pelos instrumentos de intervenção do BCB sobre a volatilidade cambial é identificada através do modelo proposto por Baba, Engle, Kraft e Kroner (BEKK), do Dynamic Conditional Correlation (DCC) e do Constant Conditional Correlation (CCC). Estes modelos consideram que a volatilidade varia no tempo, permitindo identificar qual a influência que os instrumentos utilizados pelo BCB exerceram sobre a volatilidade do câmbio em cada período. O modelo $\operatorname{BEKK}(q, p, k)$ pode ser formalizado como segue:

$$
\begin{gathered}
\varepsilon=H_{t}^{\frac{1}{2}} \mathbf{v}_{t} \\
H_{t}=A A^{\prime}+\sum_{i=1}^{q} A_{i} \varepsilon_{t-i} \varepsilon_{t-i}^{\prime} A_{i}^{\prime} \sum_{j=1}^{p} B_{j} H_{t-j} B_{j}{ }^{\prime}
\end{gathered}
$$

Em que $C, A$ e $B$ são matrizes kxk de parâmetros e $C$ é uma matriz triangular superior com p e q sendo as ordens das matrizes do modelo e $\mathrm{k}$ é o número de séries utilizado.

A limitação apresentada pelo modelo $B E K K$ é que conforme o número de variáveis cresce o número de parâmetros cresce rapidamente. Além disto, A e B não possuem interpretação direta. Uma alternativa ao modelo BEKK é o modelo de correlação condicional constante $(C C C)$ proposto por Bollerslev (1990), o qual é obtido a partir da decomposição de Cholesky $\left(H=L_{t} G L_{t}\right)$ da matriz de covariâncias:

$$
H_{t}=\alpha_{\mathrm{o}}+\alpha_{1} \varepsilon_{t-1}^{2}+\beta_{1} H_{t-1}
$$

sendo $\alpha_{1}$ e $\beta_{1}$ matrizes não-negativas definidas, $\varepsilon_{t-1}^{2}$ pode ser reescrito como:

$$
\varepsilon_{t}^{2}=\alpha_{\mathrm{o}}+\left(\alpha_{1}+\beta_{1}\right) \varepsilon_{t-1}^{2}+n_{t}-\beta_{1} n_{t}
$$

que é um ARMA(1,1) bivariado com $\mathrm{n}_{\mathrm{t}}=\varepsilon_{\mathrm{t}-1}^{2}-\mathrm{H}_{\mathrm{t}}$. A grande limitação apresentada pelo $C C C$ é que ele considera que a matriz de correlação entre os 
GIOVANINI, A.; MEURER, R. A Influência Exercida pelos Instrumentos de Intervenção do Banco Central do Brasil....

ativos permanece constante ao longo do tempo. Tse e Tsui (2002), propõem o modelo de correlação condicional dinâmica $(D C C)$, o qual contorna esta limitação: $H=D_{t} R_{t} D_{t}$, em que D é uma matriz diagonal com GARCH univariados e $\mathrm{R}_{\mathrm{t}}$ é uma matriz de correlação dos resíduos padronizados:

$$
R_{t}=\left(1-\theta_{1}-\theta_{2}\right) R_{t}+\theta_{1} \varphi_{t-1}+\theta_{2} R_{t-1}
$$

sendo $\theta_{1}$ e $\theta_{2}$ parâmetros não-negativos sujeitos à restrição $\theta_{1}+\theta_{2}<1$ e $\varphi$ uma matriz positiva definida:

$$
\varphi_{i j t-1}=\frac{\sum_{m=1}^{M} u_{i t-m}-u_{j t-m}}{\sqrt{\left(\sum_{m=1}^{M} u_{i t-m}^{2}\right)\left(\sum_{m=1}^{M} u_{j t-m}^{2}\right)}},
$$

em que $u_{i t}=\varepsilon_{i t} / h_{i t}$.

Engle e Sheppard (2001) propuseram um modelo semelhante: $H=D_{t} R_{t} D_{t}$ no qual $\mathrm{D}_{\mathrm{t}}$ é definido como uma matriz diagonal com $G A R C H$ univariados, enquanto $R_{t}$ é uma matriz de correlação dos resíduos padronizados com dinâmica:

$$
Q_{t}=\left(1-\sum_{l=1}^{L} a_{l}\right)-\left(\sum_{s=1}^{S} \beta_{s} \sum_{l=1}^{L} a_{l}\right) u_{t-1} u_{t-1}^{\prime}+\sum_{s=1}^{S} \beta_{s} Q_{t-s}
$$

em que Q é matriz de variância não condicional, na qual u, $a_{l}$ e $\beta_{s}$ são parâmetros não negativos que satisfazem a restrição $\sum_{l=1}^{L} a_{l}+\sum_{s=1} \beta_{s}<1$ (Tse e Tsui, 2002).

A significância estatística dos modelos estimados é avaliada através do forecast encompassing test. Segundo Harvey et al. (1997), um conjunto de previsões incorpora (encompasses) um conjunto de previsões concorrente, se este último não contribui para uma redução estatisticamente significativa do erro quadrático médio de previsão quando combinado com o conjunto de previsões original.

$$
e_{1 t}=\alpha y_{2 t}+\varepsilon_{t}
$$

Em que $\boldsymbol{e}_{1 t}$ e $\boldsymbol{e}_{2 t}$ são os erros do modelo 1 e do modelo 2, respectivamente, e $y_{1 t}$ e $y_{2 t}$ são as previsões destes modelos. O forecast encompassing test testa a hipótese nula, Ho: $\alpha=0$, de que o modelo 2 não possui informação 
GIOVANINI, A.; MEURER, R. A Influência Exercida pelos Instrumentos de Intervenção do Banco Central do Brasil.... adicional em relação ao modelo 1 (Burckhardt, 2012).

Dada a crise de 2007 e 2008, realizam-se testes para identificar se ela provocou uma quebra estrutural nas variáveis analisadas. A existência de quebra estrutural pode levar a interpretações inadequadas em relação à significância dos parâmetros estimados (Gregory e Hansen, 1996). O primeiro teste realizado é a estimação recursiva dos parâmetros:

$$
\begin{gathered}
Y_{t}=\sum_{j=1}^{k} \beta_{j} j_{j, t}+\varepsilon, \quad \mathrm{t}=1, \ldots, \mathrm{T} \\
\varepsilon_{t} \approx^{i i d}\left(N, \sigma^{2}\right)
\end{gathered}
$$

A estimação recursiva permite identificar e acompanhar o comportamento dos parâmetros, mostrando-se útil na construção e avaliação de regressões. Se a estimativa dos parâmetros se estabiliza conforme o tamanho da amostra aumenta há evidências de que não há quebra estrutural no modelo estimado (Young, 2011).

O segundo teste realizado é a soma cumulativa dos resíduos padronizados CUSUM, $W(k)$, proposto por Brown, Durbin e Evans (1975):

$$
W(k)=\frac{1}{\hat{\sigma}} \sum_{i-h+1}^{N} \varepsilon_{n}(i) \operatorname{com} \sigma^{2}=\frac{1}{N-h} \sum_{i-h+1}^{N} \varepsilon_{n}^{2}(i)=\frac{S(N)}{N-h},
$$

Em que, $\varepsilon_{n}(i)$ é obtido para cada ponto i e h é o número de parâmetros estimados. Se as somas cumulativas violarem os limites de probabilidade $\mathrm{h}, \mp \mathrm{a}(\mathrm{N}-\mathrm{h})^{0,5}$ e N. $\pm 3 \mathrm{a}(\mathrm{N}-\mathrm{h})^{0,5}$, em que a é um coeficiente dado por Brown, Durbin e Evans (1975) para diferentes níveis de confiança, em qualquer ponto, há evidência de instabilidade nos parâmetros. $\mathrm{O}$ terceiro teste realizado é o teste do quadrado da soma cumulativa dos resíduos (CUSUMSQ)

$$
S(k)=\frac{1}{S(N)} \sum_{i=h+1}^{k} \varepsilon_{n}^{2}(i)
$$

o qual identifica se a variância do termo de erro está mudando ao longo do tempo. A limitação apresentada pelo teste CUSUM e pelo teste CUSUMSQ é que eles são sensíveis a qualquer tipo de mudança no modelo (Young, 2011). 
GIOVANINI, A.; MEURER, R. A Influência Exercida pelos Instrumentos de Intervenção do Banco Central do Brasil....

Os testes de Dickey-Fuller aumentado (ADF) e KPSS foram utilizados para verificar a estacionariedade das séries. Caso as séries não sejam estacionárias, a literatura recomenda a diferenciação das mesmas, $d_{t}=X_{t}-X_{t-1}$. A diferenciação permite que as séries se tornem estacionárias, porém leva à perda de informação relevante, pois o nível da variável é importante. A metodologia desenvolvida por Toda e Yamamoto (1995) representa uma alternativa ao modelo VAR tradicional e permite contornar esta limitação que a diferenciação da taxa de juro impõe nos resultados obtidos. Toda e Yamamoto (1995) propuseram a estimação de um modelo com uma defasagem adicional e mostraram que os parâmetros estimados possuem distribuição normal, independente de as variáveis serem estacionárias, integradas ou cointegradas.

A seção 5 a seguir apresenta os resultados das estimações. Três modelos VAR alternativos foram estimados. O primeiro é o modelo VAR, proposto na equação (1), que considera que o câmbio é explicado pelo câmbio defasado e pelos instrumentos utilizados pelo BCB. O segundo utiliza as mesmas variáveis, mas adota a metodologia proposta por Toda e Yamamoto (1995). Por fim, estima-se um terceiro modelo VAR o qual considera que o câmbio é explicado pelo câmbio defasado, pelo desvio do câmbio em relação às expectativas dos agentes e pelo desvio da taxa de juros em relação às expectativas dos agentes. Além dos modelos VAR, se recorre ao modelo BEKK para estimar a volatilidade condicional do câmbio.

\section{Resultados}

A Tabela 2 apresenta as estatísticas descritivas relativas às variáveis utilizadas $^{2}$. Durante o período analisado, o câmbio flutuou entre R \$/US\$1,53 e R \$/ US\$ 2,86, sendo que a sua cotação média foi de 1,97 R \$/US\$. A menor taxa de juros Selic observada foi de $7,11 \%$ e a maior foi de $13,67 \%$, sendo que a taxa média de juros foi de 10,44\%. O teste ADF indica que todas as variáveis são estacionárias em nível. O KPSS indica que todas as variáveis analisadas, inclusive a taxa de juros e o câmbio são estacionárias. O teste para a presença de quebra estrutural indica que não ocorreu quebra estrutural nos parâmetros, todavia o teste CUSUMSQ indica que a variância do termo de erro muda ao longo do tempo ${ }^{3}$.

2 Todos os resultados apresentados nesta seção foram gerados no software MatLab, as únicas exceções são os testes dos parâmetros recursivos, CUSUM e CUSUMSQ, que foram gerados no Eviews. 3 Para mais detalhes ver as seções 5.1 e 5.2 
GIOVANINI, A.; MEURER, R. A Influência Exercida pelos Instrumentos de Intervenção do Banco Central do Brasil....

TABELA 2 - ESTATÍSTICAS DESCRITIVAS DAS VARIÁVEIS - 14/12/2006 E $02 / 12 / 2014$

\begin{tabular}{l|c|c|c|c|c|c|c|c|c}
\hline $\begin{array}{l}\text { Vari- } \\
\text { ável }\end{array}$ & Min & Max & Média & $\begin{array}{c}\text { Desvio } \\
\text { padrão }\end{array}$ & $\begin{array}{c}\text { Curto- } \\
\text { se }\end{array}$ & $\begin{array}{c}\text { Assi- } \\
\text { me- } \\
\text { tria }\end{array}$ & ADF & KPSS & $\begin{array}{c}\text { Que- } \\
\text { bra } \\
\text { estru- } \\
\text { tural }\end{array}$ \\
\hline $\mathrm{S}_{\mathrm{t}}$ & 1,53 & 2,86 & 1,97 & 0,27 & 2,48 & 0,54 & $-27,05^{* *}$ & $24,97^{* *}$ & Não \\
$\mathbf{i}_{\mathrm{t}}$ & 7,11 & 13,67 & 10,44 & 1,76 & 2,21 & $-0,25$ & $-25,35^{* *}$ & $3.88^{* *}$ & Não \\
$\mathrm{I}_{t}^{\text {Vist }}$ & $-39,05$ & 63,40 & $-5,47$ & 11,39 & 7,78 & 1,05 & $9,82^{* *}$ & $0,75^{* *}$ & Não \\
$\mathrm{I}_{\mathrm{t}}^{\text {Swap }}$ & 0 & $9.76 \mathrm{E}+3$ & 414.13 & $1.10 \mathrm{n}+03$ & 20.96 & 3.81 & $-13,41^{* *}$ & $5,53^{* *}$ & Não \\
$\mathrm{I}_{\mathrm{t}}^{\text {Swap* }}$ & $-7.3 \mathrm{e}+03$ & 0 & -79.09 & 460.03 & 82.20 & -8.12 & $-21,81^{* *}$ & $0,31^{* *}$ & Não \\
$\mathrm{S}_{\mathrm{t}, \mathrm{e}}$ & 1,25 & 2,70 & 1,96 & 0,27 & 2,27 & 0,48 & $-2,19^{*}$ & $11,64^{*}$ & Não \\
$\mathrm{i}_{\mathrm{t} . \mathrm{e}}$ & 7,30 & 14,30 & 10,58 & 1,78 & 2,27 & $-0,21$ & $-1,12^{*}$ & $27,56^{*}$ & Não \\
$\mathrm{i}_{t-1}^{e}$ & $-1,41$ & 0,86 & 0,08 & 0,42 & 3.15 & $-0,47$ & $-2,78^{* *}$ & $4,80^{* *}$ & Não \\
$\mathrm{S}_{t-1}^{e}$ & $-0,54$ & 0,19 & $-0,01$ & 0,05 & 19,82 & $-2,34$ & $-10,97^{* *}$ & $0,65^{* *}$ & Não \\
\hline
\end{tabular}

Fonte: Elaboração própria, ${ }^{*}$ após diferenciar, ${ }^{*} *$ sem diferenciar.

Na Figura 1 encontram-se consolidados os dados referentes à evolução diária da taxa de câmbio, da taxa de juros, intervenções no mercado a vista e swap cambial. O câmbio apresentou uma tendência de valorização no período 20062008 , atingindo o piso de $1,56 \mathrm{R} \$ / \mathrm{US} \$$ em 01/o8/2008. A maior aversão ao risco, apresentada pelos investidores estrangeiros em decorrência da crise financeira internacional provocou uma depreciação do câmbio, que atingiu o pico de 2,50 R \$/US\$ em 05/12/2008. A partir de então, a taxa de câmbio volta a apresentar uma tendência de apreciação, a qual só é revertida em 09/11/2009, 1,70 R \$/US\$. Desta data até 25/05/2010 o câmbio se apreciou, chegando a 1,88 R \$/US\$ e reverteu, alcançando o piso de 1,55 R $\$ / \mathrm{US} \$$ em 01/o8/2011. A moeda brasileira apresentou tendência de depreciação a partir de então, alcançando o pico de 2,86 em 02/02/2015. 
FIGURA 1 - EVOLUÇÃO DIÁRIA DO CÂMBIO, JUROS E INTERVENÇÕES CAMBIAIS.
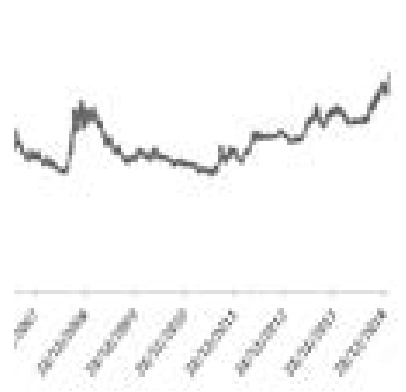

Figura 1.1: 5 ,

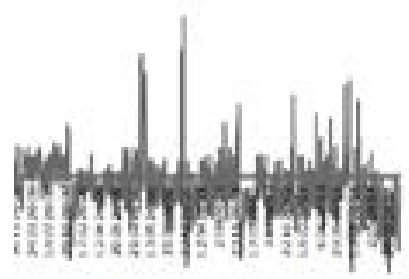

Figura 1.3: $\mathrm{i}_{\mathrm{f}}^{\text {lis }}$

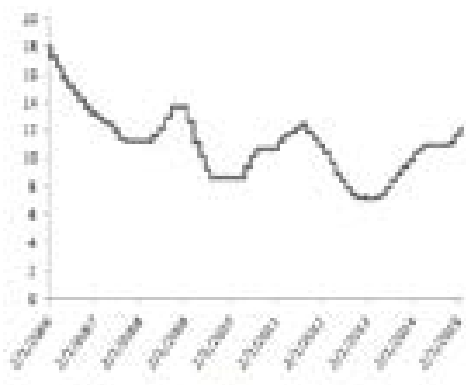

Figura 1.2; $\mathrm{i}_{1}$

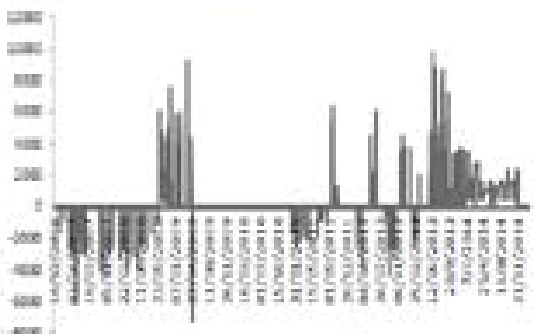

Figura 1.4: $\mathrm{i}_{1}^{\text {Swa }}$

Fonte: Elaboração própria.

Durante o período analisado a taxa de juros apresentou três movimentos de queda e três movimentos de alta, em 2008, em 2011 e em 2013. O aumento da taxa de juros, em 2008, evidencia que o BCB recorreu a este instrumento para intervir no câmbio, buscando diminuir os efeitos da crise sobre o câmbio.

Durante o período analisado, a maior parte das intervenções realizadas pelo BCB no mercado a vista buscaram diminuir a oferta de dólar no mercado doméstico. O BCB teve que intervir aumentando a oferta de dólar no mercado para fazer frente ao aumento na demanda apenas em três períodos específicos: durante a crise financeira internacional (09/10/2008 a 30/01/2009), quando houve uma forte depreciação do Real frente ao Dólar, no período 05/06/2013-11/06/2013 e no período 24/06/2013-28/06/2013. Estas intervenções ocorreram devido à sinalização do Federal Reserve dos EUA de que passaria a adotar uma política de contração monetária. 
GIOVANINI, A.; MEURER, R. A Influência Exercida pelos Instrumentos de Intervenção do Banco Central do Brasil....

As intervenções via swap apresentam dois clusters, os quais indicam que o BCB recorreu a este instrumento para diminuir a volatilidade cambial e abrandar as expectativas do mercado, principalmente no período 2008/2009.

\subsection{Modelo sem expectativas}

Os resultados consolidados na Tabela 3 indicam que apenas $\Delta \mathrm{S}_{\mathrm{t}-}, \mathrm{I}_{\mathrm{t}-1}^{\text {Swap }} \mathrm{e}$ $\mathrm{IOF}_{\mathrm{t}-1}$ explicam o retorno do câmbio. O resultado encontrado para o teste de Breusch-Pagan $(0,17)$ evidencia que o modelo estimado não é autocorrelacionado e o valor encontrado para o coeficiente de determinação, o,oo6, indica que o modelo estimado possui um baixo poder de explicação.

TABELA 3 - RESULTADOS DO MODELO VAR PARA O CÂMBIO, SEM EXPECTATIVAS E SEM A APLICAÇÃO DA METODOLOGIA PROPOSTA POR TODA E YAMAMOTO, PERÍODO 14/12/2006-30/08/2013.

\begin{tabular}{|c|c|c|}
\hline Variável \Data & Parâmetros & Desvio Padrão \\
\hline$\beta_{0}$ & -0.0011 & 0.0011 \\
\hline$\Delta \mathrm{S}_{\mathrm{t}-1}$ & $-0.0590^{* *}$ & 0.0226 \\
\hline $\mathrm{I}_{\mathrm{t}-1}^{\mathrm{Visit}}$ & $1.27 \mathrm{OE}-\mathrm{O} 5$ & $2.140 \mathrm{O}-\mathrm{O} 5$ \\
\hline $\mathrm{I}_{\mathrm{t}-1}^{\text {Swap }}$ & $4.15 \mathrm{oE}-\mathrm{O} 7^{*}$ & 2.270E-O7 \\
\hline $\mathrm{I}_{\mathrm{t}-1}^{\mathrm{Swap}}$ & $-3.940 \mathrm{O}-08$ & 5.200E-O7 \\
\hline$\Delta \mathrm{i}_{\mathrm{t}-1}$ & 0.0055 & 0.0092 \\
\hline$I O F 1_{t-1}$ & $0.0004^{*}$ & 0.0006 \\
\hline$I O F 2_{t-1}$ & 0.0011 & 0.0006 \\
\hline $\mathrm{R}^{2}$ & 0,006 & \\
\hline Breusch-Pagan & $0,17^{\#}$ & \\
\hline
\end{tabular}

Fonte: Elaboração própria, ${ }^{*}$ significativo com um nível de confiança de $90 \%,{ }^{* *}$ significativo com um nível de confiança de 95\%, "Não rejeita a hipótese de ausência de autocorrelação.

A presença de quebra estrutural é identificada através da estimação recursiva dos parâmetros, cujos resultados se encontram consolidados na Figura 2. Os 
GIOVANINI, A.; MEURER, R. A Influência Exercida pelos Instrumentos de Intervenção do Banco Central do Brasil.... resultados apresentados nesta figura mostram que não ocorreu uma quebra estrutural no câmbio e na taxa de juros no segundo trimestre de 2008, os valores estimados estão dentro do intervalo de confiança.

FIGURA 2 - ESTIMAÇÃO RECURSIVA DOS PARÂMETROS, CUSUM E CUSUMSQ
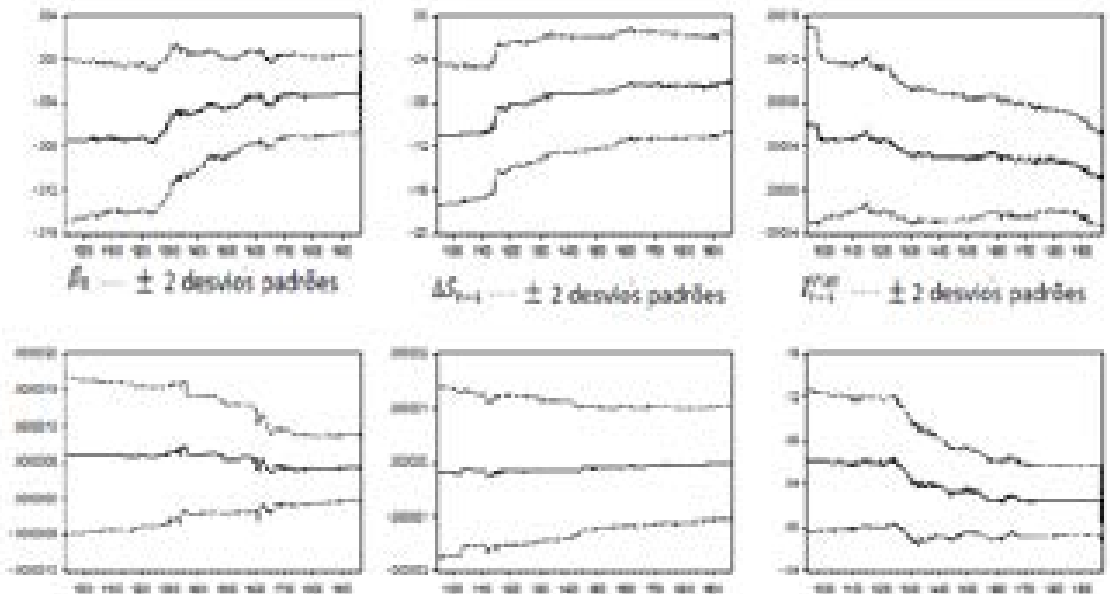

Lew +2 enviós padyes
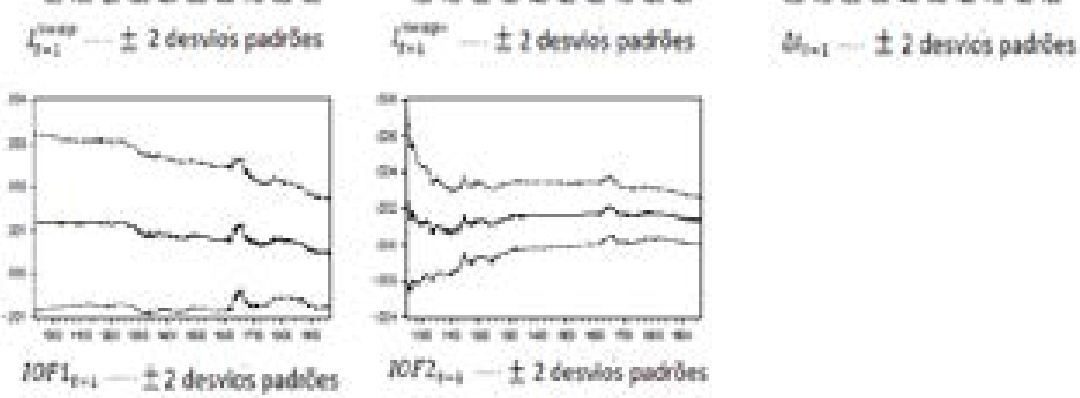

Fonte: Elaboração própria

Na Figura 3 encontra-se o valor obtido para os parâmetros recursivos, para o teste CUSUM e para o teste CUSUMSQ. O teste CUSUM mostra que não ocorreu nenhuma quebra estrutural nas variáveis que compõem o modelo. O CUSUMSQ mostra que a variância do resíduo aumentou sensivelmente. 
GIOVANINI, A.; MEURER, R. A Influência Exercida pelos Instrumentos de Intervenção do Banco Central do Brasil....

FIGURA 3 - RESULTADOS ENCONTRADOS PARA O CUSUM E PARA O CUSUMSQ
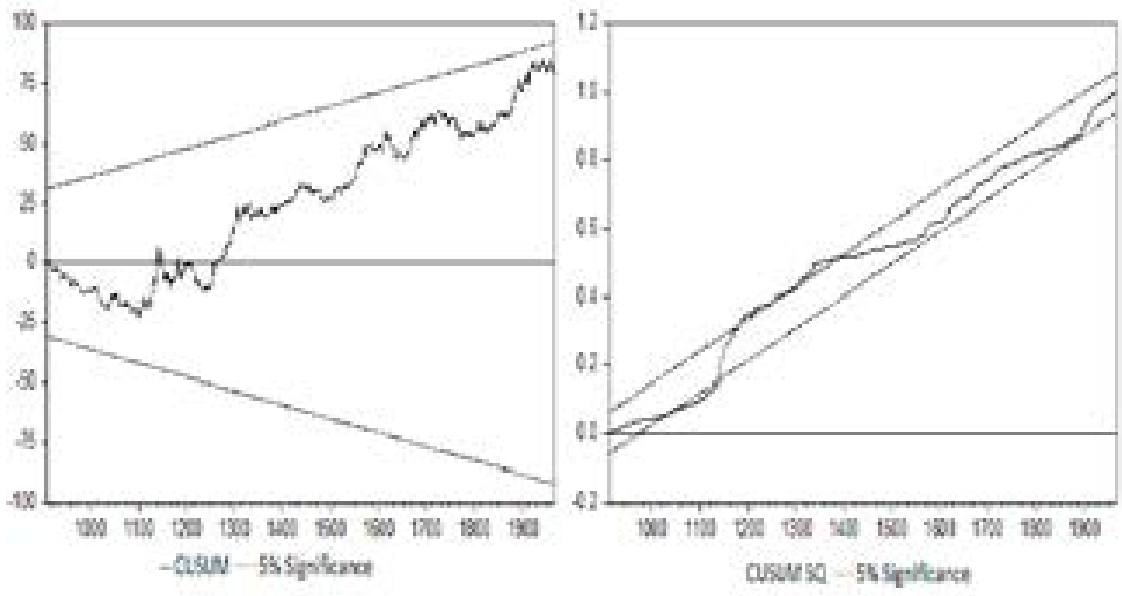

Fonte: Elaboração própria

Na Tabela 4 se encontram consolidados os resultados obtidos para o modelo VAR original, sem a aplicação da metodologia proposta por Toda e Yamamoto e para o modelo VAR com uma defasagem a mais, conforme sugerido por Toda e Yamamoto (1995). O teste de Breusch-Pagan não rejeita a hipótese de que os resíduos dos modelos estimados não são autocorrelacionados. Como o Teste CUSUMSQ, Figura 2, indica que a variância do resíduo aumentou sensivelmente entre 2008 e 2010, o modelo foi dividido em dois períodos, 14/12/2006- 01/04/2008 e 02/04/2008- 02/12/2014. 
GIOVANINI, A.; MEURER, R. A Influência Exercida pelos Instrumentos de Intervenção do Banco Central do Brasil....

TABELA 4 - RESULTADOS ENCONTRADOS PARA OS MODELOS VAR SEM EXPECTATIVAS.

\begin{tabular}{|c|c|c|c|c|c|c|c|c|}
\hline \multirow[b]{2}{*}{ Período } & \multicolumn{4}{|c|}{ Modelo Original } & \multicolumn{4}{|c|}{$\begin{array}{l}\text { Regressão com uma defasagem } \\
\text { a mais, metodologia de Toda e } \\
\text { Yamamoto (1995) }\end{array}$} \\
\hline & $\begin{array}{l}14 / 12 / 20 \\
01 / 04 / 2\end{array}$ & $\begin{array}{l}\text { oo6- } \\
008\end{array}$ & $\begin{array}{r}02 / 04 / 2 \\
02 / 12 / 2 \\
\end{array}$ & $\begin{array}{l}2008- \\
2014 \\
\end{array}$ & $\begin{array}{l}14 / 12 / 2 \\
01 / 04 / 2\end{array}$ & $\begin{array}{l}\text { oo6- } \\
2008 \\
\end{array}$ & $\begin{array}{c}02 / 04 / 20 \\
02 / 12 / 2 \\
\end{array}$ & $\begin{array}{l}008- \\
014 \\
\end{array}$ \\
\hline $\begin{array}{l}\text { Variável } \\
\text { Data }\end{array}$ & Parâmetros & $\begin{array}{l}\text { Desvio } \\
\text { padrão }\end{array}$ & Parâmetros & $\begin{array}{l}\text { Desvio } \\
\text { padrão }\end{array}$ & Parâmetros & $\begin{array}{l}\text { Desvio } \\
\text { padrão }\end{array}$ & Parâmetros & $\begin{array}{l}\text { Desvio } \\
\text { padrão }\end{array}$ \\
\hline$\beta_{\mathrm{o}}$ & -0.0008 & 0.0064 & $-0.0010^{* *}$ & 0.0012 & -0.0008 & 0.0063 & -0.0026 & 0.0017 \\
\hline$\Delta \mathrm{S}_{\mathrm{t}-1}$ & -0.0755 & 0.057 & -0.0579 & 0.0245 & -0.0759 & 0.0572 & $-0.055543^{*}$ & 0.0245 \\
\hline$\Delta \mathrm{S}_{\mathrm{t}-2}$ & - & - & - & -0.0262 & 0.0573 & 0.0573 & 0.0245 & 0.024 \\
\hline $\begin{array}{l}\mathrm{I}_{\mathrm{t}-1}^{\mathrm{V} 1 \mathrm{st}} \\
\mathrm{I}_{\mathrm{t}-2}^{\mathrm{Vist}}\end{array}$ & -0.0001 & 0.0001 & $6.72 \mathrm{E}-06$ & $\begin{array}{c}2.30 \mathrm{E}- \\
05 \\
-\end{array}$ & $\begin{array}{r}-0.0002 \\
6.5283\end{array}$ & $\begin{array}{l}0.0002 \\
0.0001\end{array}$ & $\begin{array}{l}-8.63 \mathrm{E}-06 \\
4.46 \mathrm{E}-05\end{array}$ & $\begin{array}{l}4.46 \mathrm{E}- \\
055 \\
4.45 \mathrm{E}-\end{array}$ \\
\hline $\mathrm{I}_{\mathrm{t}-1}^{\text {Swap }}$ & $\mathrm{O}$ & $\mathrm{O}$ & $\begin{array}{c}4.0133 \mathrm{e}^{-} \\
07^{*}\end{array}$ & $\begin{array}{c}2.55 \mathrm{E}- \\
07\end{array}$ & $-4.00 E-07$ & $\begin{array}{c}6.37 \mathrm{E}- \\
07\end{array}$ & $2.82 \mathrm{E}-\mathrm{O} 7$ & $\begin{array}{c}2.81 \mathrm{E}- \\
07\end{array}$ \\
\hline $\mathrm{I}_{\mathrm{t}-2}^{\text {Swap }}$ & - & - & & - & 7.79E-08 & $\begin{array}{c}6.65 \mathrm{E}- \\
07\end{array}$ & $2.86 \mathrm{E}-\mathrm{O} 7$ & $\begin{array}{c}2.85 \mathrm{E}- \\
07\end{array}$ \\
\hline $\begin{array}{l}\text { Swap* } \\
\text { t } 1\end{array}$ & - & - & $\mathrm{O}$ & $\begin{array}{c}7.14 \mathrm{E}- \\
07\end{array}$ & - & - & $7.15 \mathrm{E}-07$ & $\begin{array}{c}7.12 \mathrm{E}- \\
07\end{array}$ \\
\hline $\mathrm{I}_{\mathrm{t}-2}^{\text {Swap* }}$ & - & - & - & & - & - & $7.15 \mathrm{E}-07$ & $\begin{array}{c}7.15 \mathrm{E}- \\
07\end{array}$ \\
\hline$\Delta \mathrm{i}_{\mathrm{t}-1}$ & -0.0039 & 0.058 & 0.0057 & 0.0095 & - & - & - & - \\
\hline $\mathrm{i}_{\mathrm{t}-1}$ & - & - & - & - & 0.0003 & 0.0008 & 0.0007 & 0.0015 \\
\hline $\mathrm{i}_{\mathrm{t}-2}$ & - & - & - & - & -0.0004 & 0.0006 & -0.0005 & 0.0015 \\
\hline $\mathrm{IOF}_{\mathrm{t}-1}$ & - & - & - & - & - & - & 0.0143 & 0.0143 \\
\hline $\mathrm{IOF} 1_{\mathrm{t}-2}$ & & & - & - & - & - & 0.0143 & 0.0143 \\
\hline $\mathrm{IOF} 2_{\mathrm{t}-1}$ & - & - & 0.0011 & 0.0011 & - & - & $0.0106^{*}$ & 0.0107 \\
\hline $\mathrm{IOF} 2_{\mathrm{t}-2}$ & & - & & - & - & - & $0.0106^{*}$ & 0.0106 \\
\hline $\mathrm{R}^{2}$ & 0,15 & - & 0,22 & - & 0,32 & - & 0,31 & - \\
\hline $\begin{array}{l}\text { Breusch- } \\
\text { Pagan }\end{array}$ & $14^{\#}$ & - & 228,14 & - & $14,88^{\#}$ & - & 69,98 & - \\
\hline
\end{tabular}

Fonte: Elaboração própria, "Significativa a 90\%, " Não rejeita a hipótese de ausência de autocorrelação.

O resultado encontrado para o modelo VAR estimado segundo a metodologia proposta por Toda e Yamamoto (1995), Tabela 4, indica que no período 14/12/2006-01/04/2008 nenhum dos coeficientes estimados é significativo e no período 02/04/2008-02/12/2014 o coeficiente estimado para $\mathrm{I}_{\mathrm{t}-1}$ Swap é significativo.

O teste Encompasing indica que, para o período 14/12/2006-01/04/2008, o modelo VAR estimado com a metodologia proposta por Toda e Yamamoto (1995) possui melhor ajuste, $\alpha=-2,74 \mathrm{e}-09$ do que o modelo VAR tradicional 
GIOVANINI, A.; MEURER, R. A Influência Exercida pelos Instrumentos de Intervenção do Banco Central do Brasil.... com a taxa de juros diferenciada. No período 02/04/2008-02/12/2014, o teste Encompasing indica que o modelo estimado com a metodologia proposta por Toda e Yamamoto (1995) possui um ajuste pior que o modelo VAR tradicional, estimado com a taxa de juros diferenciada, $\alpha=-4,84 \mathrm{e}-\mathrm{O}, 04$.

\subsection{Resultados encontrados para o modelo com expecta-}

A Figura 4 apresenta o valor do teste de quebra estrutural, para a estimação recursiva dos parâmetros, o qual indica que não houve quebra estrutural.

FIGURA 4 - RESULTADOS ENCONTRADOS PARA A ESTIMAÇÃO RECURSIVA DOS PARÂMETROS - COM EXPECTATIVAS
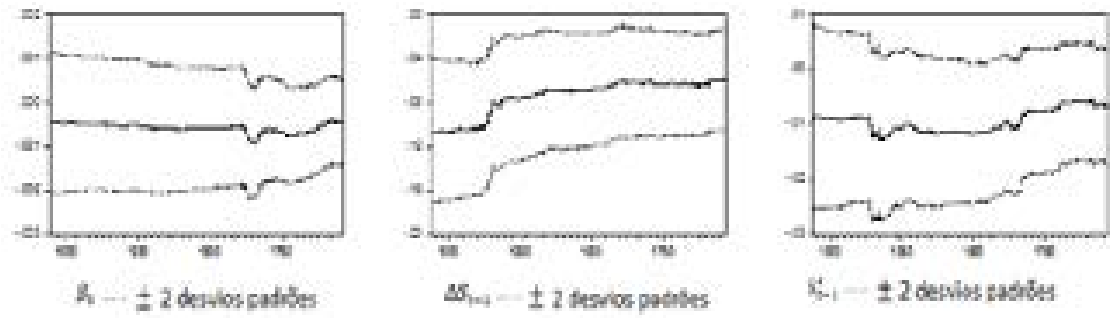

$\Delta_{\text {hes }} \pm 2$ fesvor pathles

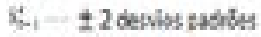
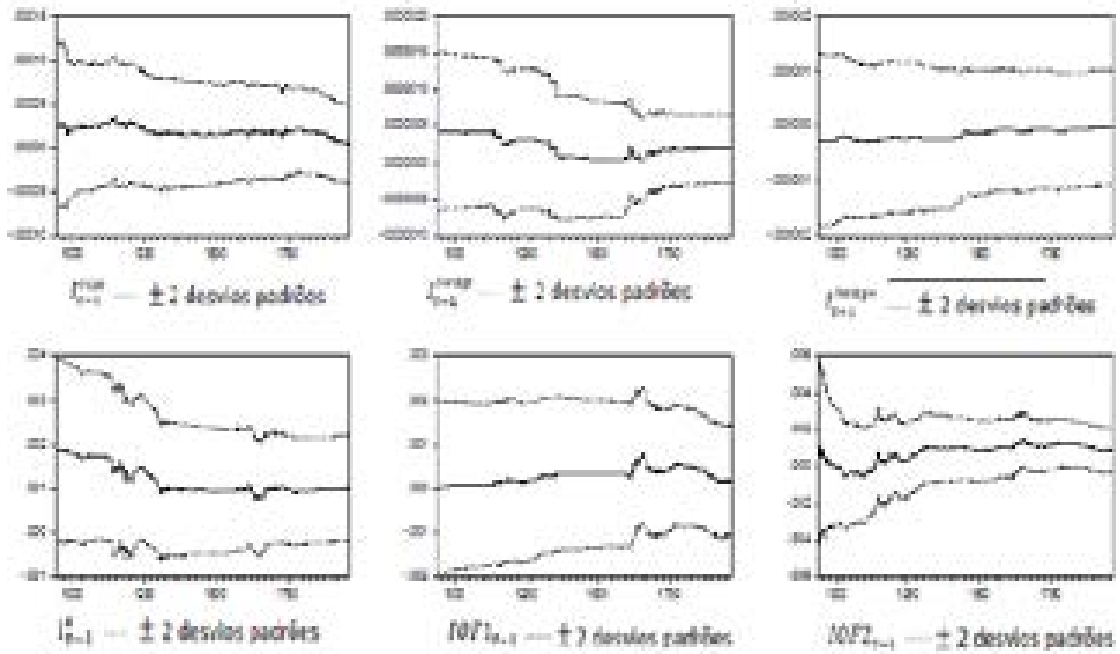

Fonte: Elaboração própria.

A Figura 5 mostra os resultados obtidos para o teste CUSUM e CUSUMSQ. O teste CUSUM indica que não ocorreu quebra estrutural nos parâmetros e 
GIOVANINI, A.; MEURER, R. A Influência Exercida pelos Instrumentos de Intervenção do Banco Central do Brasil....

o CUSUMSQ indica que ocorreu uma quebra estrutural durante o período analisado. Como o CUSUMSQ indica que a variância do resíduo aumentou sensivelmente, foram estimados três modelos VAR para os seguintes períodos: 14/12/2006 - 28/05/2009, 29/05/2009 - 12/01/2012 e 13/01/2012 $-02 / 12 / 2014$.

FIGURA 5 - RESULTADOS ENCONTRADOS PARA O CUSUM E PARA O CUSUMSQ - COM EXPECTATIVAS

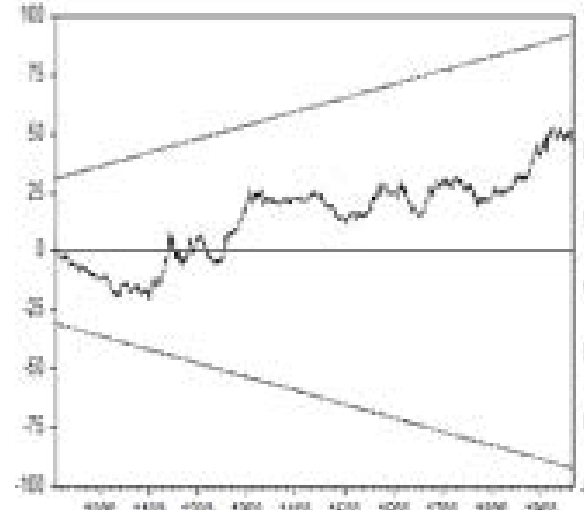

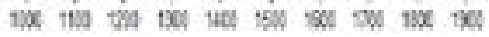

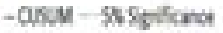

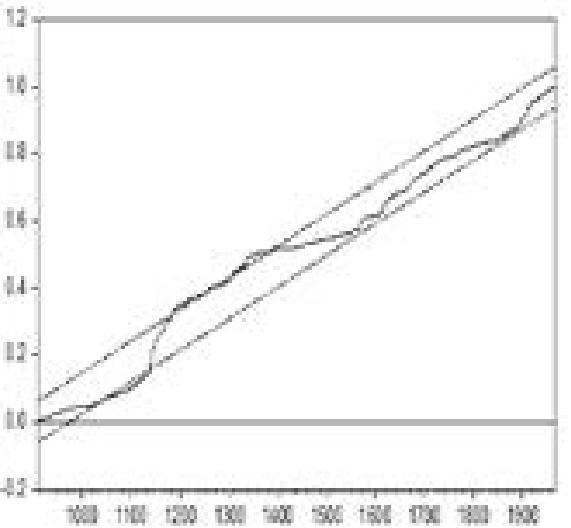

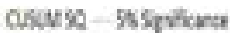

Fonte: Elaboração própria.

A Tabela 5 apresenta os valores estimados para o modelo VAR. No período 14/12/2006 - 02/12/2014, apenas $\Delta \mathrm{St}-1, \mathrm{~S}_{\mathrm{t}-1}^{\mathrm{e}} \mathrm{e}_{\mathrm{t}-1}^{\mathrm{e}}$ são significativos para um nível do confiança de $95 \%$. 
GIOVANINI, A.; MEURER, R. A Influência Exercida pelos Instrumentos de Intervenção do Banco Central do Brasil....

TABELA 5 - RESULTADOS DOS MODELOS VAR COM EXPECTATIVAS COM O RETORNO DO CÂMBIO COMO VARIÁVEL DEPENDENTE, PERÍODO 14/12/2006$02 / 12 / 2014$.

\begin{tabular}{|c|c|c|c|c|c|c|c|c|}
\hline $\begin{array}{l}\text { V a r i } \\
\text { á vel } \\
\text { Data }\end{array}$ & \multicolumn{2}{|c|}{$\begin{array}{c}14 / 12 / 2006- \\
02 / 12 / 2014\end{array}$} & \multicolumn{2}{|c|}{$\begin{array}{l}14 / 12 / 2006- \\
28 / 05 / 2009\end{array}$} & \multicolumn{2}{|c|}{$\begin{array}{c}\text { 29/05/2009 - } \\
12 / 01 / 2012\end{array}$} & \multicolumn{2}{|c|}{$\begin{array}{l}\text { 13/01/2012 - } \\
02 / 12 / 2014\end{array}$} \\
\hline $\begin{array}{l}\text { Discri- } \\
\text { mina- } \\
\text { ção }\end{array}$ & $\begin{array}{l}\text { Parâme- } \\
\text { tros }\end{array}$ & $\begin{array}{l}\text { Desvio } \\
\text { padrão }\end{array}$ & $\begin{array}{c}\text { Parâme- } \\
\text { tros }\end{array}$ & $\begin{array}{l}\text { Desvio } \\
\text { padrão }\end{array}$ & $\begin{array}{c}\text { Parâme- } \\
\text { tros }\end{array}$ & $\begin{array}{l}\text { Desvio } \\
\text { padrão }\end{array}$ & $\begin{array}{c}\text { Parâme- } \\
\text { tros }\end{array}$ & $\begin{array}{l}\text { Desvio } \\
\text { padrão }\end{array}$ \\
\hline$\beta_{\mathrm{o}}$ & -0.0001 & 0.0003 & -0.0003 & 0.0007 & $\begin{array}{c}2.752 \mathrm{E}- \\
\mathrm{O} 5\end{array}$ & 0.0005 & -0.0003 & 0,001 \\
\hline$\Delta \mathrm{S}_{\mathrm{t}-1}$ & -0.0600 & 0.0225 & $\begin{array}{c}-0.1363 \\
87^{* *}\end{array}$ & 0.0406 & $0.067^{*}$ & 0.0389 & 0.0107 & 0.0372 \\
\hline $\mathbf{S}_{t-1}^{e}$ & -0.0100 & 0.0051 & -0.0106 & 0.0104 & 0.0006 & 0,009 & 0.0049 & 0.0072 \\
\hline$I_{t-1}^{\text {Vist }}$ & $\begin{array}{l}-7.239 \mathrm{E}- \\
06\end{array}$ & $2.168 \mathrm{E}-$ & $\begin{array}{c}-6.227 \mathrm{E}- \\
05\end{array}$ & $8.845^{\mathrm{E}-}$ & 2.796E- & $\begin{array}{c}2.517 \mathrm{E}- \\
05\end{array}$ & $\begin{array}{c}-3.260 \mathrm{E}- \\
05\end{array}$ & $\begin{array}{c}-2.573 \mathrm{E}- \\
05\end{array}$ \\
\hline $\mathrm{I}_{\mathrm{t}-1}^{\mathrm{Swap}}$ & $\begin{array}{l}1.951 \mathrm{E}- \\
07 \\
-3.685 \mathrm{E}-\end{array}$ & $\begin{array}{l}2.232 \mathrm{E}- \\
\mathrm{O} 7 \\
5.18 \mathrm{oE}-\end{array}$ & $\begin{array}{c}9.224 \mathrm{E}- \\
\text { o7 } \\
-1.409 \mathrm{E}-\end{array}$ & $\begin{array}{l}6.233 \mathrm{E}- \\
\text { O7 } \\
9.055^{\mathrm{E}-}\end{array}$ & $\begin{array}{l}1.609 \mathrm{E}- \\
06 \\
-5.959 \mathrm{E}-\end{array}$ & $\begin{array}{c}1.030 \mathrm{OE}- \\
06 \\
-1.28 \mathrm{oE}-\end{array}$ & $\begin{array}{c}4.549 \mathrm{E}- \\
\text { o8 } \\
5.975 \mathrm{E}-\end{array}$ & $\begin{array}{c}2.289 \mathrm{E}- \\
07 \\
7.465 \mathrm{E}-\end{array}$ \\
\hline & 08 & 07 & 08 & 07 & 07 & 06 & 08 & 07 \\
\hline$i_{t-1}^{e}$ & $0.001^{*}$ & 0.0006 & $0.003^{*}$ & 0.0015 & 0.0001 & 0,0011 & -0.0002 & 0.0010 \\
\hline $\mathrm{IOF} 1_{\mathrm{t}-1}$ & -0.0002 & 0.0005 & - & - & -0.0004 & 0,0015 & - & - \\
\hline $\mathrm{IOF} 2_{\mathrm{t}-1}$ & & & - & - & - & - & 0,0012 & 0.0007 \\
\hline $\mathrm{R}^{2}$ & 0,84 & - & 2.68E-01 & - & o,o3 & - & $\begin{array}{c}4.28 \mathrm{E}- \\
01\end{array}$ & - \\
\hline ch-Pa- & $0,10^{\#}$ & - & $2,38 \#$ & - & $1,24 \#$ & - & $1,33 \#$ & - \\
\hline
\end{tabular}

Fonte: Elaboração própria, ${ }^{,}$Significativa a 95\%," Não rejeita a hipótese de ausência de autocorrelação.

Os valores encontrados para o teste de estacionariedade de Dickey-Fuller para $\mathrm{i}_{t}^{e}$ e $\mathrm{s}_{t}^{e},-11.38$ e -3.89, respectivamente, indicam que as séries são estacionárias, não sendo necessário diferenciá-las.

Os resultados obtidos para o modelo VAR, Tabela 5 , indicam que no período 14/12/2006 - 28/05/2009 apenas $\mathrm{S}_{\mathrm{t}-1}$ e $\mathrm{i}_{\mathrm{t}-1}{ }^{\mathrm{e}}$ explicam o retorno do câmbio. No período 29/05/2009 - 12/01/2012 o coeficiente estimado para $\mathrm{S}_{\mathrm{t}-1}$ é significativo para um nível de confiança de 99\% e no período 13/01/2012 - 02/12/2014 apenas $\mathrm{S}_{\mathrm{t}-1} \mathrm{e}_{\mathrm{t}-1}{ }^{\text {Vist }}$ são significativos. 
GIOVANINI, A.; MEURER, R. A Influência Exercida pelos Instrumentos de Intervenção do Banco Central do Brasil....

Para o primeiro e o segundo períodos os resultados indicam que a variação de um real em $\Delta \mathrm{S}_{\mathrm{t}-1}$ resulta em um retorno do câmbio de R $\$ / \mathrm{US} \$-0,14$ e R \$/ US\$-0,067, respectivamente. Um desvio em $1 \%$ de $\mathrm{i}_{\mathrm{t}-1}^{\mathrm{e}}$ leva a uma variação de -0,004 unidades monetárias no retorno do câmbio.

Os resultados encontrados para o modelo com expectativas evidenciam que a intervenção do BCB no câmbio através da taxa de juros, $\mathrm{i}_{\mathrm{t}-1}^{\mathrm{e}}$, no período 14/12/2006 - 28/05/2009 influenciou no retorno do câmbio. Este resultado vai em sentido oposto aos resultados encontrados por Oliveira e Plaga (2011), segundo o qual na crise de 1999 o BCB não conseguiu influenciar a volatilidade cambial através da taxa de juros. A explicação para isto é que a economia brasileira se encontrava em expansão, apresentando taxa média de crescimento do PIB, 4,5\% no período 2004-2008. As elevadas taxas de crescimento da economia brasileira contribuíram para que, com o advento da crise, as incertezas dos investidores estrangeiros em relação à economia brasileira não aumentassem ( $\mathrm{S}_{t-1}^{\mathrm{e}}$ é significativo, indicando um aumento da incerteza dos investidores). Os investidores internacionais continuaram respondendo às mudanças na taxa de juros. Uma evidência favorável a este argumento é a forte apreciação do câmbio observada em 2009.

Conforme indicado por Meurer et al. (2007) e Viola (2009), os resultados encontrados indicam que $\mathrm{I}_{\mathrm{t}-1}$ Swap influenciou no retorno apresentado pelo câmbio tanto no modelo estimado através da metodologia proposta por Toda e Yamamoto (1995), período 02/04/2008-02/12/2014, quanto no modelo que leva em consideração as expectativas dos agentes, período 14/12/2006 $-30 / 11 / 2014$.

Os resultados encontrados para o IOF são contraditórios. O modelo estimado através da metodologia proposta por Toda e Yamamoto (1995) indica que este instrumento influenciou no retorno do câmbio e o modelo que leva em consideração as expectativas dos agentes indica que o $\mathrm{IOF}_{\mathrm{t}-1}$ influenciou no comportamento do câmbio, no período 13/01/2012 - 30/o8/2013. Estes resultados contradizem a afirmação de Munhoz (2013), segundo o qual o IOF não foi capaz de influenciar na trajetória do câmbio.

Segundo Oliveira e Plaga (2011) e Meurer et al. (2007), o BCB consegue influenciar o comportamento do câmbio através de $\mathrm{I}_{\mathrm{t}-1}{ }^{\text {Vist }}$. Os resultados encontrados para o modelo com expectativas não confirmam estas afirmações, no período 13/01/2012 - 30/o8/2013 as intervenções do BCB via $\mathrm{I}_{\mathrm{t}-1}{ }^{\text {Vist }}$ influenciaram na trajetória do câmbio.

Como o modelo que possui o câmbio e a taxa de juros esperados como argumentos possui melhor ajuste, estimam-se as volatilidades condicionais com base no resíduo gerado por este modelo. O teste encompassing foi utilizado para identificar qual o modelo ( $D C C, C C C$ ou $B E K K)$ que possui melhor poder de explicação sobre a volatilidade do câmbio. O valor encontrado para o teste $t$, para os erros dos modelos DCC e CCC estimados em função da volatilidade 
GIOVANINI, A.; MEURER, R. A Influência Exercida pelos Instrumentos de Intervenção do Banco Central do Brasil....

apresentada pelo modelo BEKK foram: -4,5508 e -4,0013 para o período 14/12/2006 - 28/05/2009; -5,4093, e -5,9018 para o período 29/05/2009 - 12/01/2012 e -6,2517 e -6,6396 para o período 13/o1/2012 - 02/12/2014, indicando que o BEKK é o modelo que melhor explica a volatilidade.

Na Figura 8 se encontra consolidado o resultado encontrado para a volatilidade condicional, obtidos através do modelo BEKK, para os períodos 14/12/2006 - 02/12/2014. As correlações estimadas para $i_{t}$ e $I_{t}^{\text {Swap }}$ variam bastante ao longo do tempo. Por outro lado, a volatilidade estimada para $\mathrm{s}_{t-1}^{\mathrm{e}}$ no período 14/12/2006 - 28/05/2008, 29/05/2008 - 12/01/2012 e 13/01/2012 - 02/12/2014 apresenta uma variação considerável apenas em períodos específicos, evidenciando a formação de agrupamentos. Por fim, a volatilidade condicional estimada para $\mathrm{I}_{\mathrm{t}-1}^{\text {Vist }}$ no período 29/05/2008 02/12/2014 evidencia a formação de um agrupamento específico durante a crise do subprime, em 2008, a partir de 2011 a volatilidade condicional apresenta uma variação considerável.

FIGURA 9 - CORRELAÇÃO CONDICIONAL, PERÍODO 13/01/2012 - 02/12/2014
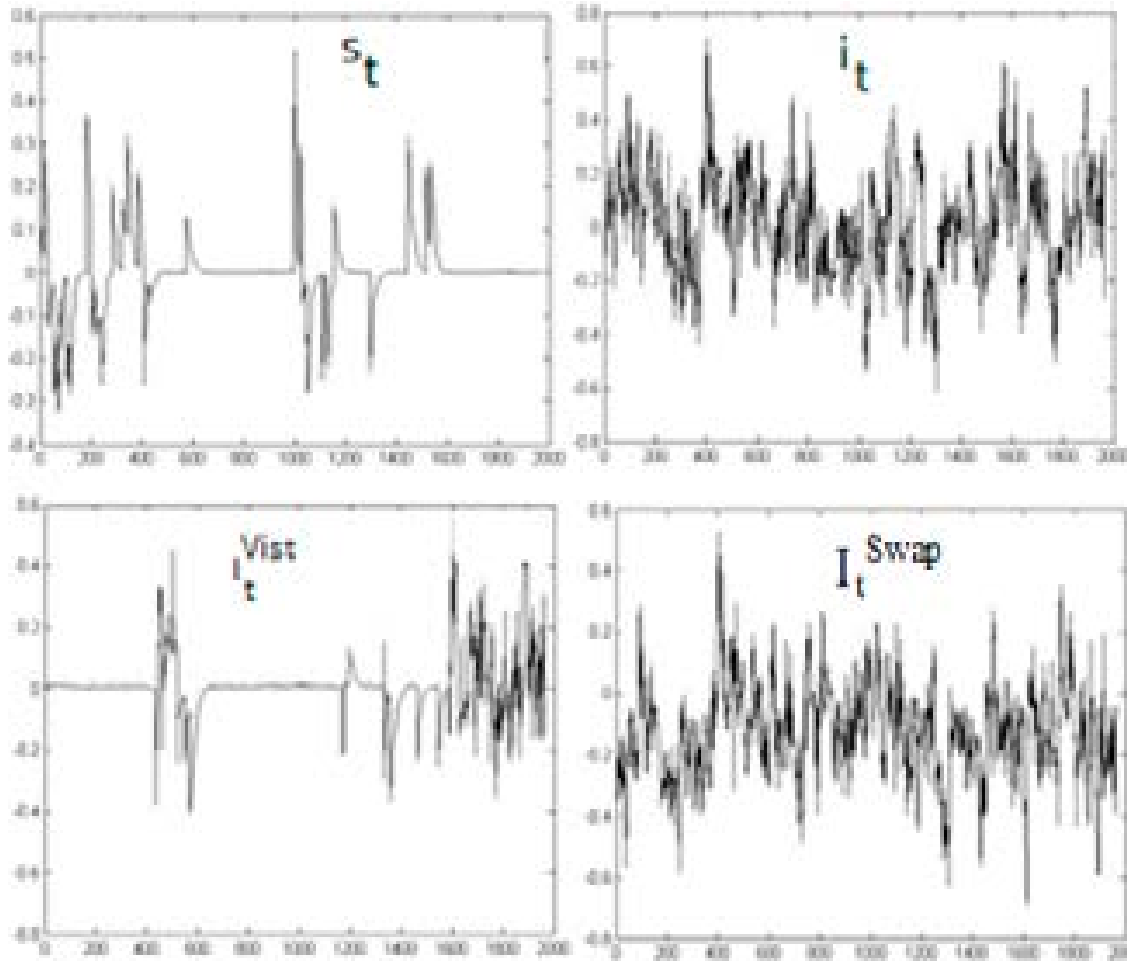

Fonte: Elaboração Própria 
GIOVANINI, A.; MEURER, R. A Influência Exercida pelos Instrumentos de Intervenção do Banco Central do Brasil....

Os resultados encontrados evidenciam a impossibilidade de se extrair conclusões reducionistas sobre o modo como os instrumentos utilizados pelo BCB influenciam no câmbio. Isto é explicado porque, em primeiro lugar, o BCB não utilizou os instrumentos de modo sistemático, recorreu a eles de forma discricionária, utilizando o instrumento que julgava mais adequado a cada contexto econômico. Em segundo lugar, a influência exercida por cada um dos instrumentos utilizados sobre a trajetória do câmbio variou, mostrando-se sensível ao contexto econômico. Estes fatores foram captados pelos resultados encontrados, uma vez que a significância, em termos estatísticos, dos instrumentos utilizados variou consideravelmente de um período para outro.

\section{Considerações Finais}

Os resultados encontrados para o teste de quebra estrutural não descartam a possibilidade de ter ocorrido quebra estrutural no segundo trimestre de 2008. Os coeficientes recursivos estimados para o modelo que tem o câmbio defasado e a taxa de juros como variáveis explicativas mostram evidências de que ocorreu uma quebra estrutural nos parâmetros para estas duas variáveis, enquanto que o teste CUSUMSQ mostra a presença de quebra estrutural em ambos os modelos estimados.

Os resultados encontrados para o modelo VAR estimado com base nas variáveis que levam as expectativas dos agentes em consideração mostram que em todos os períodos o retorno defasado do câmbio influenciou no câmbio. Os instrumentos utilizados pelo BCB para influenciar no câmbio variaram bastante ao longo do período analisado. No período no qual ocorreu a crise do subprime, 14/12/2006 - 28/05/2009, o desvio do câmbio em relação às expectativas e o desvio da taxa de juros em relação às expectativas dos agentes influenciaram o câmbio. No período posterior a crise, 29/05/2009 12/01/2012, as intervenções via taxa de juros deixam de influenciar o câmbio e as intervenções via swap cambial passam a influenciar. Por fim, no último período analisado, 13/01/2012 - 02/12/2014, apenas as intervenções via mercado a vista influenciaram o câmbio.

Um aumento da volatilidade do câmbio significa aumento da incerteza dos agentes sobre o comportamento futuro do câmbio. O BCB utiliza as intervenções via swap cambial e mercado a vista, buscando fornecer proteção para os agentes contra a volatilidade do câmbio, com o objetivo de evitar que o aumento das incertezas resulte no aumento da volatilidade cambial.

Os resultados encontrados estão indicando que as intervenções via swap reverso, no período 14/12/2006 - 28/05/2008 e 13/01/2012 - 02/12/2014 e via 
GIOVANINI, A.; MEURER, R. A Influência Exercida pelos Instrumentos de Intervenção do Banco Central do Brasil....

mercado à vista, no período 29/05/2008 - 12/01/2012 influenciam na volatilidade do câmbio. Estes resultados vão de encontro aos resultados encontrados por Meurer et al. (2007), Viola (2009) e Oliveira e Plaga (2011), segundo os quais as intervenções no mercado a vista e via swap cambial influenciam no retorno do câmbio e o IOF não influenciou no comportamento do câmbio. A intervenção via taxa de juros não foi significativa, contrariando os resultados de Meurer et al. (2007) e Oliveira e Plaga (2011), porém o desvio da taxa de juros em relação às expectativas explicou o comportamento do câmbio.

Uma possibilidade de expansão deste trabalho é analisar mais detalhadamente o comportamento das expectativas dos agentes e do efeito das intervenções da autoridade monetária sobre as expectativas da taxa de câmbio e sua volatilidade.

\section{Referências}

Araújo, J. D., \& Goldfajn, I. (2004). Suavizando movimentos da taxa de câmbio ou adicionando volatilidade? Um estudo empírico sobre intervenções do Banco Central no mercado de câmbio. Master's thesis, Pontifícia Universidade Católica do Rio de Janeiro.[Links].

Bastos, E. K. X. \& Fontes, P. V. S. Mercado de câmbio brasileiro e intervenções do banco central. Nota Técnica: Banco Central do Brasil. URL [On line]: www.bcb. gov.br/pt-br/Paginas/bc-anuncia-programa-de-leiloes-de-cambio-22-8-2013-. aspx, acesso em: 22 abril 2014.

Brown, R. L., Durbin, J., \& Evans, J. M. (1975). Techniques for testing the constancy of regression relationships over time. Journal of the Royal Statistical Society. Series B (Methodological), 149-192.

Bollerslev, T. (1990). Modelling the coherence in short-run nominal exchange rates: a multivariate generalized ARCH model. The Review of Economics and Statistics, 498-505.

Burckhardt, N. (2012). Essays on the Correlation of Foreign Exchange Rates (Doctoral dissertation, University of St. Gallen).

Carvalho, B. S. D. M., \& Garcia, M. G. (2008). Ineffective controls on capital inflows under sophisticated financial markets: Brazil in the nineties. In Financial Markets Volatility and Performance in Emerging Markets (pp. 29-96). University of Chicago Press.

dos Santos Cerqueira, V. (2011). Volatilidade da taxa de câmbio real e taxa de juros no Brasil: Evidências de um modelo VAR-GARCH-M para o período 1999-2010 (No. 1586). Texto para Discussão, Instituto de Pesquisa Econômica Aplicada (IPEA).

Engle, R. F., \& Sheppard, K. (2001). Theoretical and empirical properties of dynamic conditional correlation multivariate GARCH (No. w8554). National Bureau of Economic Research. 
GIOVANINI, A.; MEURER, R. A Influência Exercida pelos Instrumentos de Intervenção do Banco Central do Brasil....

Gregory, A. W., \& Hansen, B. E. (1996). Residual-based tests for cointegration in models with regime shifts. Journal of econometrics, 7o(1), 99-126.

Gonçalves, C. E., \& Guimarães, B. (2011). Monetary policy, default risk and the exchange rate. Revista Brasileira de Economia, 65(1), 33-45.

Harvey, D., Leybourne, S., \& Newbold, P. (1997). Testing the equality of prediction mean squared errors. International Journal of forecasting, 13(2), 281-291.

Merlin, C. E. (2002). Uma avaliação da taxa de câmbio real de equilíbrio para o Brasil: 1984-2000.

Meurer, R., Moura, G. V., \& Nunes, M. S. (2007). O vencimento da dívida pública cambial influencia a taxa de câmbio? Um estudo econométrico para o brasil no período 2003-2004. Economia Aplicada, 11(1), 55-72.

Meurer, R., Teixeira, F. W., \& Tomazzia, E. C. (2010). Efeitos das Intervenções Cambiais a vista na Taxa de Câmbio R \$/US \$ de 1999 a 2008: Um Estudo de Evento. Revista Brasileira de Finanças, 8(2), 229-254.

Mohanty, M., \& Scatigna, M. (2005). Has globalisation reduced monetary policy independence?. BIS papers, 23, 17-58.

Munhoz, V. D. C. V. (2013). Vulnerabilidade externa e controle de capitais no Brasil: uma análise das inter-relações entre câmbio, fluxos de capitais, IOF, juros e risco-país. Nova Economia, 23(2), 371-402.

Nelson, D. B., \& Cao, C. Q. (1992). Inequality constraints in the univariate GARCH model. Journal of Business \& Economic Statistics, 1O(2), 229-235.

Obstfeld, M. (1990). The effectiveness of foreign-exchange intervention: Recent experience, 1985-1988. In International policy coordination and exchange rate fluctuations (pp. 197-246). University of Chicago Press.

Oliveira, F. N. D., \& Plaga, A. (2011). Eficácia das intervenções do Banco Central do Brasil sobre a volatilidade condicional da taxa de câmbio nominal. Revista brasileira de economia, 65(1), 71-92.

Rogoff, K. (1984). On the effects of sterilized intervention: An analysis of weekly data. journal of Monetary Economics, 14(2), 133-150.

Sicsú, J. (2002). Flutuação cambial e taxa de juros no Brasil. Revista de Economia Política, 22(3), 132-137.

Silva, G. J. C. D., \& Resende, M. F. D. C. (2010). Eficácia dos controles de capitais no Brasil: uma abordagem teórica e empírica alternativa. Estudos Econômicos (São Paulo), 4O(3), 617-649.

Toda, H. Y., \& Yamamoto, T. (1995). Statistical inference in vector autoregressions with possibly integrated processes. Journal of econometrics, 66(1), 225-250.

De Toledo, J. E. C. (2002). Risco Brasil: o efeito-Lula e os efeitos-Banco Central. Revista de Economia Política, 22(3), 87.

Tse, Y. K., \& Tsui, A. K. C. (2002). A multivariate generalized autoregressive conditional heteroscedasticity model with time-varying correlations. Journal of Business \& Economic Statistics, 2O(3), 351-362.

Viola, A. P., Gutierrez, M. S., Lion, O. B., \& Barbedo, C. H. (2009). Impacto dos swaps cambiais na curva de cupom cambial: uma análise segundo a regressão 
GIOVANINI, A.; MEURER, R. A Influência Exercida pelos Instrumentos de Intervenção do Banco Central do Brasil.... de componentes principais (No. 198).

Young, P. C. (2011). Recursive estimation and time-series analysis: An introduction for the student and practitioner. Springer Science \& Business Media. 
